Supporting Information for:

\title{
Generation of $\mathrm{H}_{2} \mathrm{~S}$ from Thiol-Dependent NO Reactivity of Model [4Fe-4S] Cluster and Roussin's Black Anion
}

\author{
Kady M. Oakley, Ziyi Zhao, Ryan L. Lehane, Ji Ma, and Eunsuk Kim* \\ Department of Chemistry, Brown University, Providence, Rhode Island 02912, United States
}

Contents

Page

Figure S1. Set up for hydrogen sulfide detection using fluorescence sensor C7Az.

Figure S2. Calibration curve for $\mathrm{H}_{2} \mathrm{~S}$ concentration-dependent

fluorescence intensity of $\mathrm{C} 7 \mathrm{Az}$

Figure S3. Concentration-dependent EPR calibration curve for DNIC

Figure S4. Calibration curve for phenyldisulfide ( $\mathrm{PhSSPh})$

Figure S5. IR spectra of iron-nitrosyl product of $\left(\mathrm{Et}_{4} \mathrm{~N}\right)\left[\mathrm{Fe}_{4} \mathrm{~S}_{3}(\mathrm{NO})_{7}\right](\mathbf{R B A})$ react with thiol and thiolate in the presence of $\mathrm{Ph}_{3} \mathrm{CSNO}$.

Figure S6. IR spectra of iron-nitrosyl product of $\left(\mathrm{Et}_{4} \mathrm{~N}\right)\left[\mathrm{Fe}_{4} \mathrm{~S}_{3}(\mathrm{NO})_{7}\right](\mathbf{R B A})$ react with thiol and thiolate in the absence of $\mathrm{Ph}_{3} \mathrm{CSNO}$.

Figure S7. NMR spectra of iron-nitrosyl product of $\left(\mathrm{Et}_{4} \mathrm{~N}\right)\left[\mathrm{Fe}_{4} \mathrm{~S}_{3}(\mathrm{NO})_{7}\right](\mathbf{R B A})$ react with thiol and thiolate in the absence of $\mathrm{Ph}_{3} \mathrm{CSNO}$.

Figure S8. IR spectrum (in $\mathrm{KBr}$ ) of Int. A (red) resulting from $\mathbf{1} / \mathrm{NO}$ in the presence of $\mathrm{HSPh}$ and that of authentic DNIC (back). 


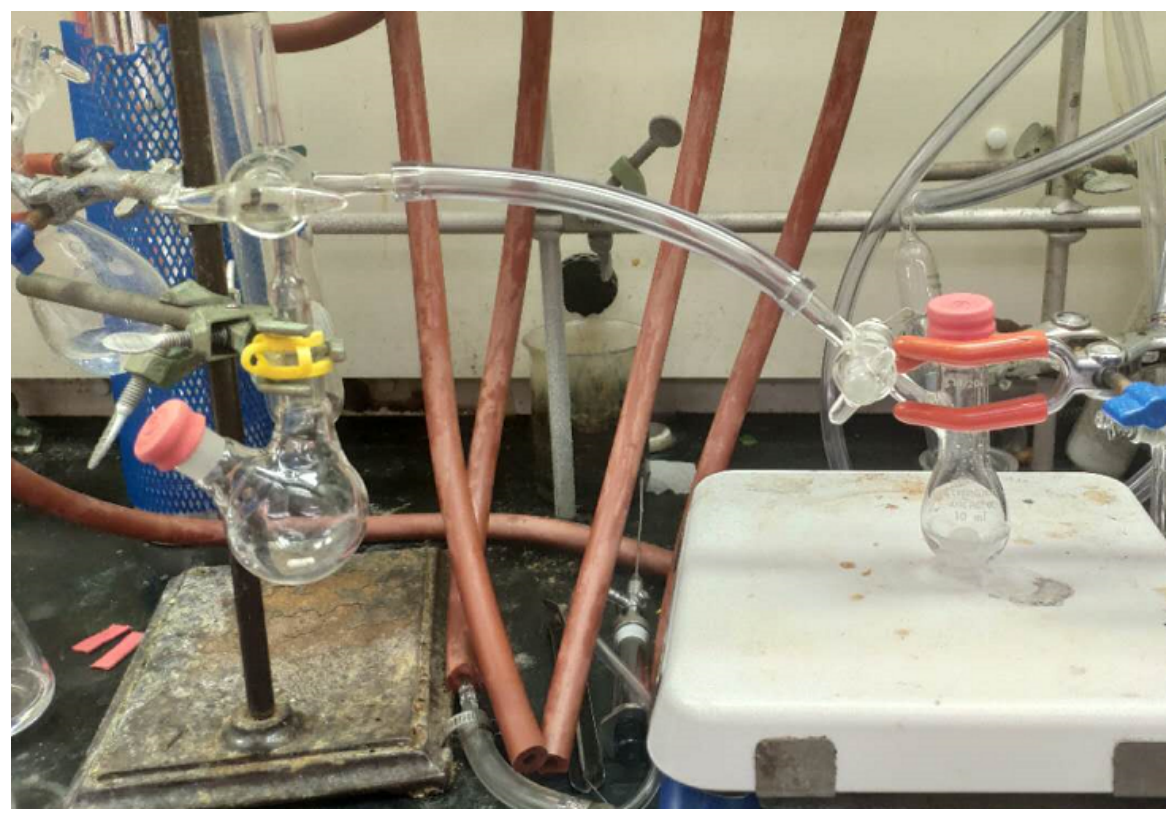

Figure S1. Set up for hydrogen sulfide detection using turn-on fluorescence sensor C7Az.

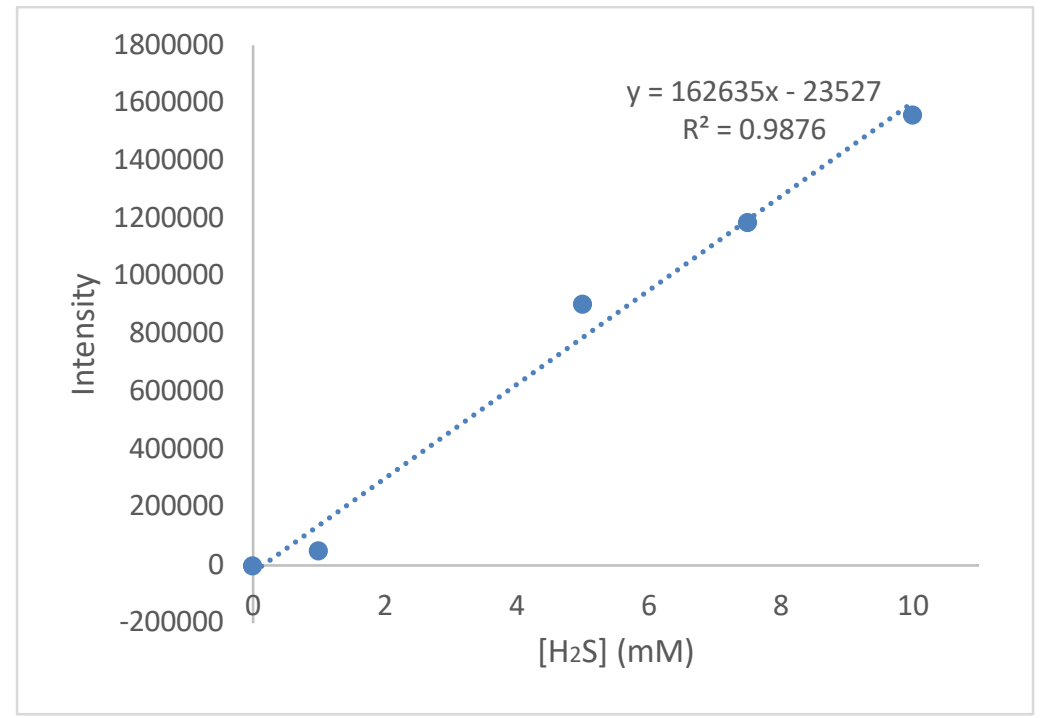

Figure S2. Calibration curve for hydrogen sulfide $\left(\mathrm{H}_{2} \mathrm{~S}\right)$ concentration-dependent fluorescence intensity of turn-on fluorescence $\mathrm{H}_{2} \mathrm{~S}$ sensor C7Az. 


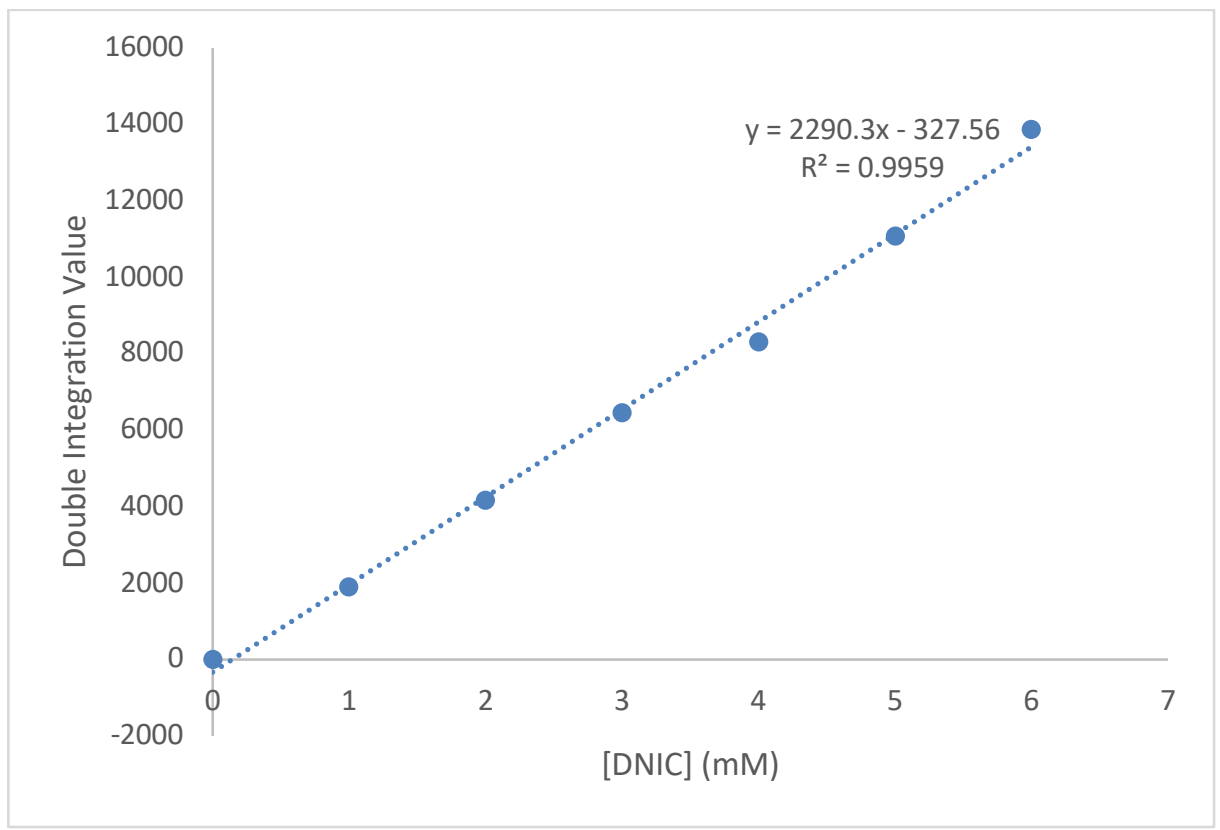

Figure S3. Concentration-dependent EPR calibration curve for DNIC at $298 \mathrm{~K}$ in MeCN. Spectra were recorded under the following conditions: microwave frequency: $9.871 \mathrm{GHz}$, microwave power: $2.0 \mathrm{~mW}$, modulation amplitude and frequency: $1.000 \mathrm{G}$ and $100 \mathrm{kHz}$.

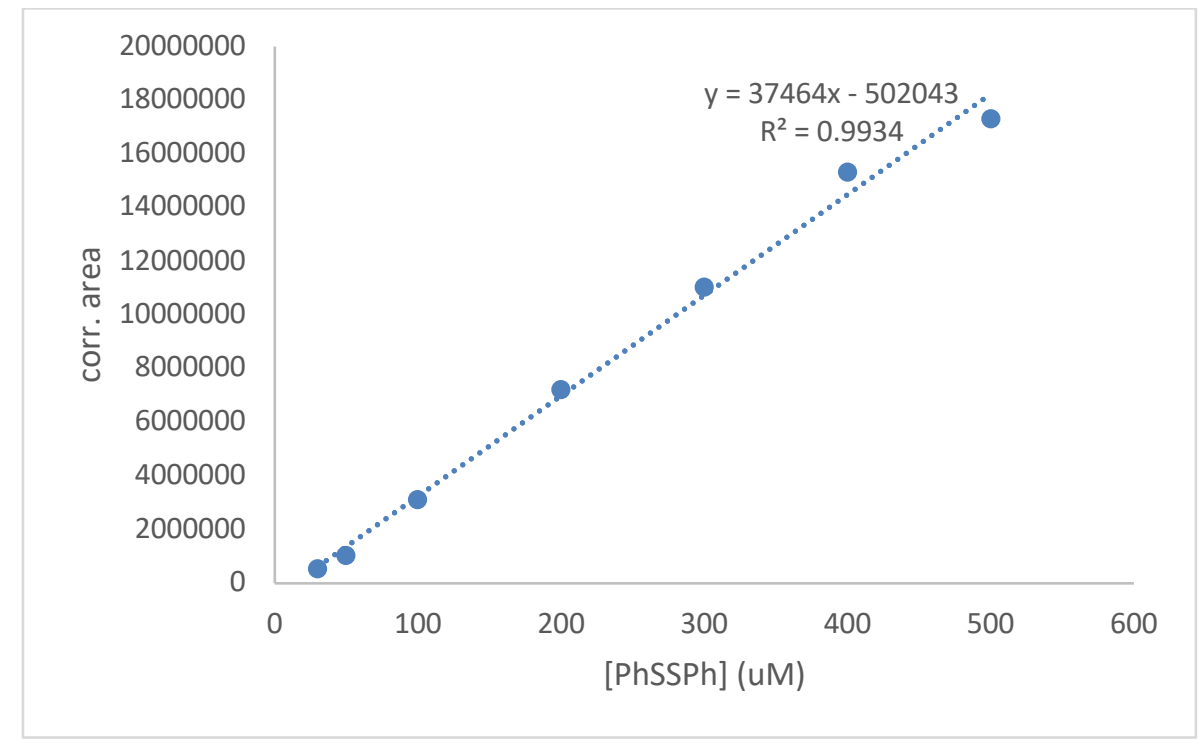

Figure S4. GC-MS calibration curve for phenyldisulfide (PhSSPh) 


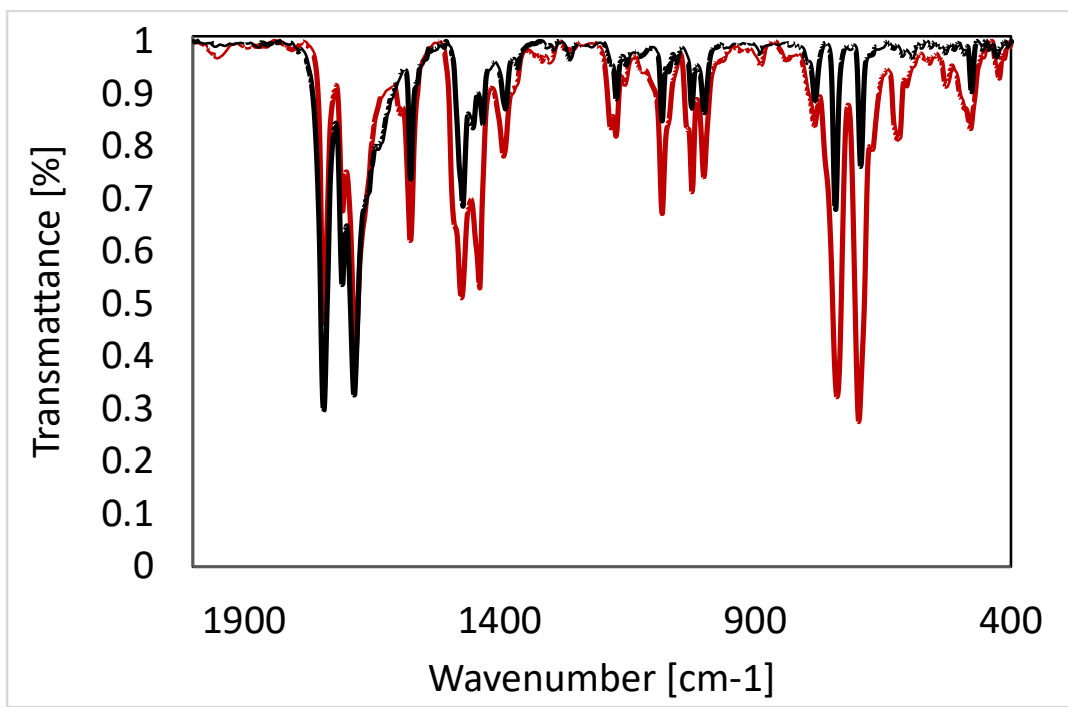

Figure S5. IR spectra $\left(\mathrm{KBr}\right.$ pellet) of the $\mathbf{v}_{\mathrm{NO}}\left(\mathrm{cm}^{-1}\right)$ region comparing $\mathrm{RBA}+10$ equiv. $\mathrm{PhSH}+$ 10 equiv. $\mathrm{Ph}_{3} \mathrm{CSNO}+20$ equiv. $\left[\mathrm{NEt}_{4}\right][\mathrm{SPh}]$ (red) to DNIC (black)

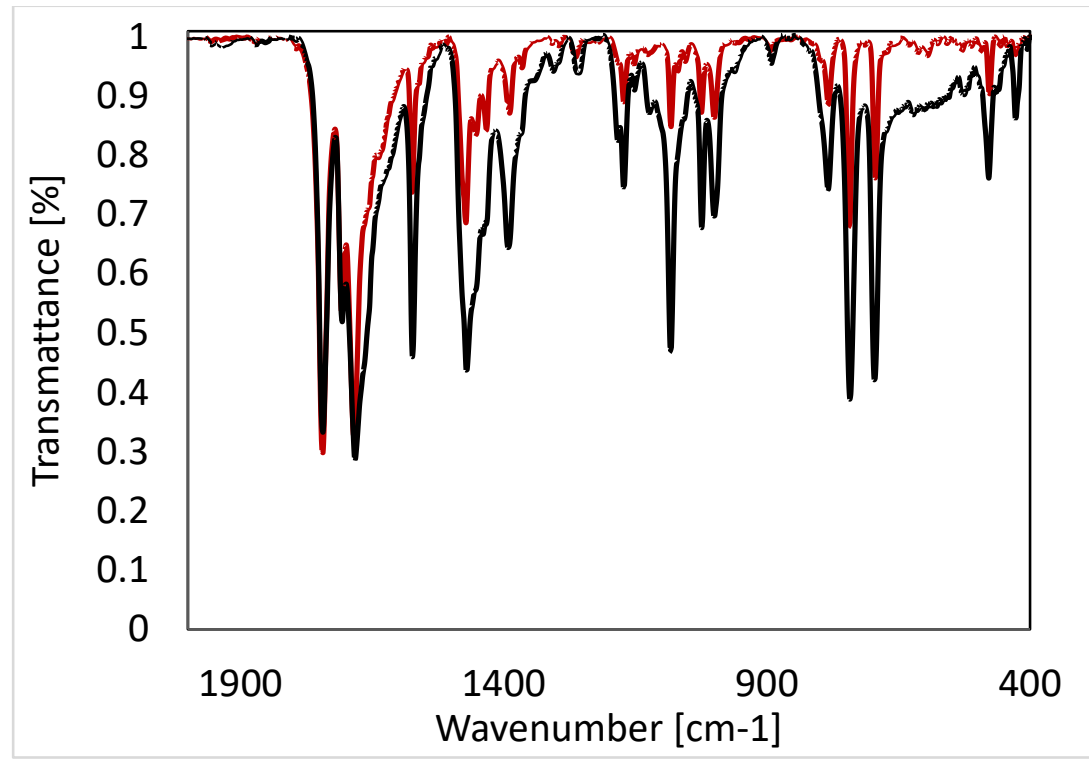

Figure S6. IR spectra $\left(\mathrm{KBr}\right.$ pellet) of the $\mathbf{v}_{\mathrm{NO}}\left(\mathrm{cm}^{-1}\right)$ region comparing $\mathrm{RBA}+10$ equiv. $\mathrm{PhSH}+$ 10 equiv. [ $\left.\mathrm{NEt}_{4}\right][\mathrm{SPh}]$ (red) to DNIC (black) 


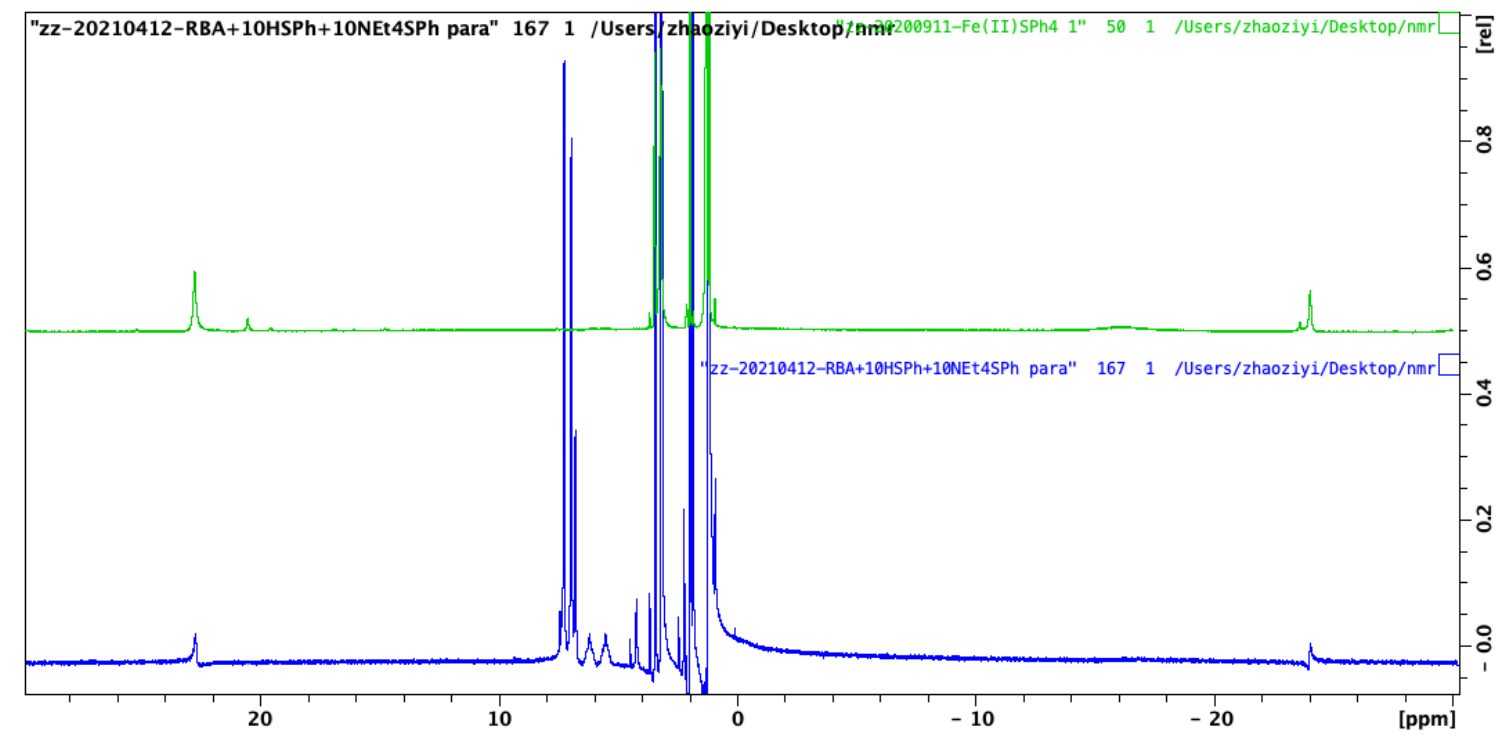

Figure S7. (blue) NMR spectra of iron-nitrosyl product of $\left(\mathrm{Et}_{4} \mathrm{~N}\right)\left[\mathrm{Fe}_{4} \mathrm{~S}_{3}(\mathrm{NO})_{7}\right](\mathbf{R B A})$ react with thiol and thiolate in the absence of PH3CSNO; (green) authentic $\left(\mathrm{NEt}_{4}\right)_{2} \mathrm{Fe}(\mathrm{II})(\mathrm{SPh})_{4}$.

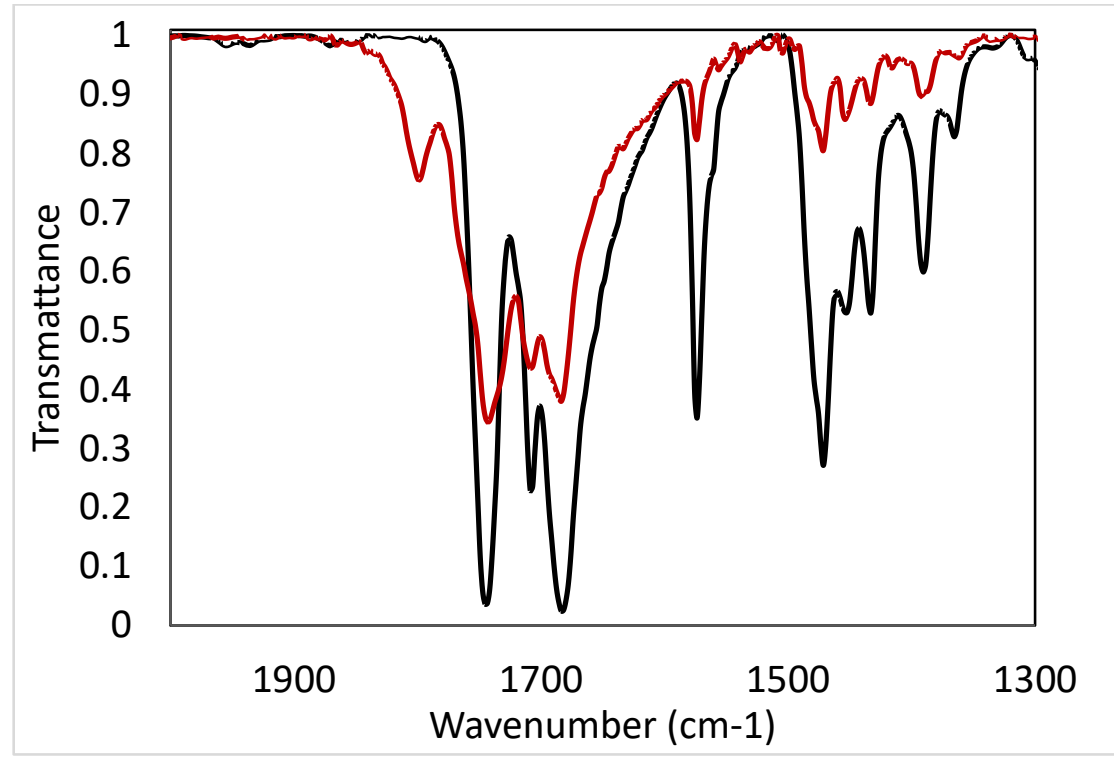

Figure S8. IR spectrum (in $\mathrm{KBr}$ ) of Int. A (red) resulting from 1/NO in the presence of HSPh and that of authentic DNIC (back). 